05

\title{
Возбуждение лазеров на парах меди прямым разрядом накопительного конденсатора через быстродействующие фототиристоры
}

\author{
(C) A.C. Кюрегян \\ ПАО „НПО Энергомодуль“, \\ 109052 Москва, Россия \\ e-mail: semlab@yandex.ru
}

Поступила в редакцию 29.08.2018 г.

В окончательной редакции 06.01.2019 г.

Принята к публикации 09.01.2019 г.

Исследована возможность применения оптопары „импульсный волоконный лазер-фототиристор“ в качестве коммутатора в схемах возбуждения лазеров на парах меди (ЛПМ). Показано, что такой коммутатор обладает наносекундным быстродействием, способен формировать монополярные и знакопеременные импульсы тока через ЛПМ с мощностью до $10 \mathrm{MW}$ и частотой повторения десятки килогерц при электрическом КПД схемы возбуждения более 95\%. Предложена простая, но весьма точная модель фототиристора, которую можно использовать в полномасштабных программах моделирования ЛПМ.

DOI: $10.21883 /$ OS.2019.04.47518.258-18

\section{Введение}

Для эффективной работы лазеров на парах меди (ЛПМ) и других металлов необходимо, чтобы схема возбуждения обеспечивала возможно более короткие (не более $10 \mathrm{~ns})$ фронты импульсов напряжения $U_{R}(t)$ на активной составляющей $R_{l}(t)$ импеданса газоразрядной трубки [1-3]. Простейшая схема возбуждения изображена на рис. 1 [1]. В качестве коммутатора в большинстве работ использовались импульсные водородные тиратроны [4], которые обладают рядом недостатков [1,5]. Вопервых, типичная предельная скорость нарастания тока тиратронов $d J / d t \approx 4 \mathrm{~A} / \mathrm{ns} \ll U_{C 0} / L \approx 50 \mathrm{~A} / \mathrm{ns} \quad\left(U_{C 0}-\right.$ начальное напряжение на накопительной емкости $C$, $L$ - индуктивность разрядного контура), а характерное время спада напряжения на тиратроне при включении порядка нескольких десятков наносекунд. Поэтому длительность фронта импульса возбуждения определяется не только параметрами схемы, но и в значительной степени свойствами тиратрона. Во-вторых, потери в тиратронах достигают $(0.4-0.6) C U_{C 0}^{2} / 2[5]$, что существенно снижает практический КПД всего устройства. В-третьих, ресурс их работы (порядка $1000 \mathrm{~h}$ ) недостаточен для ряда практических применений. Поэтому на протяжении уже десятков лет исследуются возможности замены тиратронов на более эффективные коммутаторы, однако без особого успеха.

Авторы недавней работы [6], анализируя причины застоя в развитии лазеров на парах металлов за последние 20 лет, пришли к выводу о том, „что выходная мощность и эффективность генерации современных промышленных лазеров ограничиваются несовершенством их систем питания“. Они возлагают надежды на использование нового типа разрядников - кивотронов [7,8], ко- торые позволяют резко сократить длительность фронта импульсов возбуждения ЛПМ и тем самым обеспечить „повышение эффективности генерации более чем на порядок при значительном росте выходной мощности“. Однако таким образом не решаются другие важные проблемы - повышение КПД и долговечности схемы возбуждения, так как, во-первых, использование кивотрона вносит весьма значительные дополнительные потери и, во-вторых, кивотрон является лишь обострителем фронта импульса, формируемого первичным коммутатором, в качестве которого приходится использовать все тот же тиратрон [6-8].

Между тем еще 38 лет назад авторы работы [9] предложили использовать для возбуждения ЛПМ коммутатор, состоящий из нескольких последовательно соединенных кремниевых фототиристоров специальной конструкции [10], управляемых импульсами излучения неодимового лазера. Однако ни экспериментальных, ни

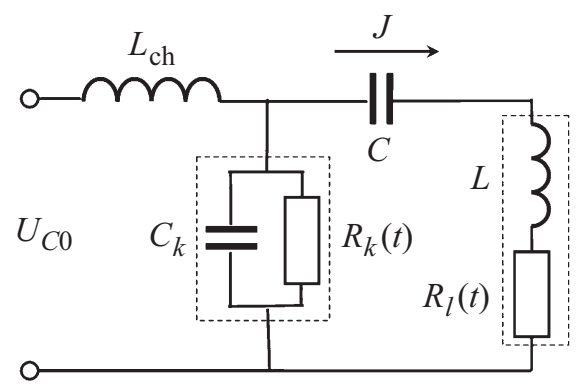

Рис. 1. Эквивалентная схема возбуждения лазера с прямым разрядом накопительной емкости $C$ через коммутатор с емкостью $C_{k}$ и переменным сопротивлением $R_{k}(t) . L_{c h}-$ индуктивность зарядного дросселя, $L-$ полная индуктивность разрядного контура, $R_{l}(t)$ - активная составляющая импеданса газоразрядной трубки лазера. 
теоретических обоснований эффективности этого технического решения в работе [9] не было представлено, и развитие подобных методов возбуждения лазеров на парах металлов приостановилось до сих пор по двум причинам. Во-первых, надежность, длительность импульсов и КПД стандартных для того времени неодимовых лазеров (типа ЛТИПЧ) были далеко недостаточны для обеспечения необходимых эксплуатационных характеристик фототиристорных коммутаторов. Вовторых, до недавнего времени фактически отсутствовала теория переключения высоковольтных структур с $p-n$ переходами из блокирующего в проводящее состояние при воздействии коротких импульсов ионизирующего облучения. Это не позволяло оптимизировать конструкцию фототиристора. Недавно такая теория была построена в работах автора [11-14], результаты которых указывают на возможность переключения высоковольтных кремниевых фототиристоров специальной конструкции в проводящее состояние за время $0.1-5 \mathrm{~ns}$ под действием импульсов света с длиной волны $\lambda \approx 1060 \mathrm{~nm}$, длительностью $t_{p h}=0.1-5 \mathrm{~ns}$, энергией $W_{p h}=0.1-1 \mathrm{~mJ}$ и частотой повторения $f$ до $80 \mathrm{kHz}$. Такие параметры управляющих импульсов легко получить с помощью современных коммерческих волоконных лазеров с практическим КПД 10-30\% [15].

Столь радикальное улучшение ситуации стимулировало нас заново проанализировать перспективность замены тиратронов на оптопару „волоконный лазерфототиристор“ в схемах возбуждения ЛПМ и других лазеров на самоограниченных переходах. Результаты этого анализа, изложенные далее, показывают, что такая оптопара является почти идеальным коммутатором, обладающим субнаносекундным быстродействием, близким к 100\% КПД и высокой долговечностью, свойственной всем твердотельным приборам.

\section{Модели ЛПМ и коммутатора}

В настоящей работе мы ограничились рассмотрением чисто электротехнической эффективности работы фототиристоров в качестве коммутаторов схемы возбуждения ЛПМ. Для решения этой задачи нет необходимости использовать полномасштабные модели газоразрядных процессов в ЛПМ, подробно описанные, например, в монографии [1]. Достаточно лишь знать правдоподобные значения индуктивности $L$ и активной составляющей импеданса газоразрядной трубки $R_{l}(t)=\Lambda / q \mu n \pi r_{p l}^{2}$, которая зависит от времени $t$ вследствие изменения подвижности $\mu$ и концентрации $n$ свободных электронов в газоразрядной плазме в процессе возбуждения $(\Lambda-$ межэлектродное расстояние газоразрядной трубки, $r_{p l}-$ эффективный радиус плазменного шнура). Мы использовали для этого предельно упрощенную нульмерную модель ЛПМ, полагая, что распределение электронов по энергии в первом приближении квазистационарно по отношению к изменению напряженности поля $E=U_{R} / \Lambda$,

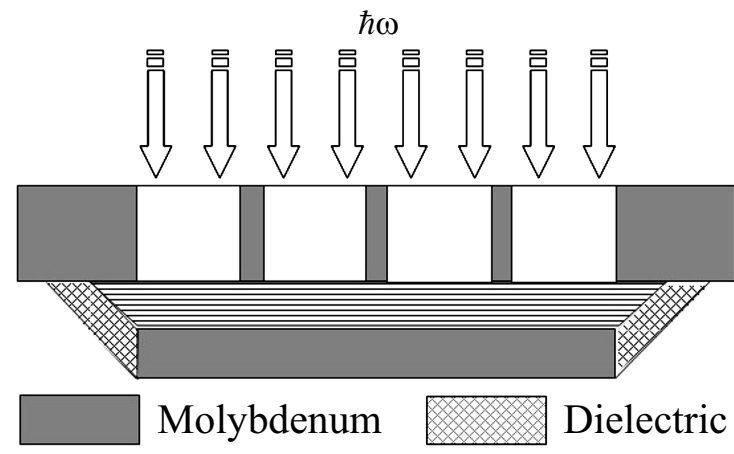

Silicon

Рис. 2. Схематичное изображение поперечного сечения фототиристора.

где $U_{R}=R_{l} J, J$ - ток контура. Если рекомбинация электронов во время импульса возбуждения пренебрежимо мала, то

$$
\begin{gathered}
R_{l}=\Lambda M(E) / q \mu_{0} n S_{p l}, \\
\frac{d n}{d t}=n\left(N_{\mathrm{Cu}}-n\right) K_{i 0} A(E), \quad n(0)=n_{0},
\end{gathered}
$$

где $n_{0}$ и $\mu_{0}$ - концентрация и подвижность свободных электронов в момент начала импульса возбуждения, $N_{\mathrm{Cu}}$ - концентрация атомов меди, $K_{i 0}$ - константа их ионизации, а $M(E)$ и $A(E)$ - мгновенные функции напряженности поля. Для них мы использовали аппроксимации

$$
M(E)=\left[1+\left(E / E_{M}\right)^{4}\right]^{1 / 16} \text { и } A(E)=\exp \left(-E_{A} / E\right),
$$

которые неплохо описывают результаты расчетов [16] при значениях $\mu_{0} N_{\mathrm{Ne}}=6.6 \cdot 10^{22} \mathrm{~B}^{-1} \mathrm{C}^{-1} \mathrm{~cm}^{-1}$, $E_{M} / N_{\mathrm{Ne}}=2.12 \cdot 10^{-12} \mathrm{~B} \cdot \mathrm{cm}^{2}, \quad K_{i 0}=3 \cdot 10^{-8} \mathrm{~cm}^{3} \mathrm{~s}^{-1}$ и $E_{A} / N_{\mathrm{Ne}}=1.36 \cdot 10^{-11} \mathrm{~V} \cdot \mathrm{cm}^{2}$.

Коммутатор представляет собой сборку из $m$ последовательно соединенных кремниевых $n^{+}-p-i-n-p^{+}$структур фототиристоров площадью $S_{k}$, помещенных между молибденовыми электродами (рис. 2). В катодном электроде и в металлизации $n^{+}$-слоя проделано множество $(\sim 100)$ окон с общей площадью $S_{0} \sim 0.5 S_{k}$ для квазиоднородного освещения. Один из возможных вариантов конструкции такого фототиристора описан в работе [17].

В блокирующем состоянии практически все напряжение $U_{k 0}$ падает на встроенных обратно смещенных $p-i-n$-диодах, толщина которых $d$ незначительно меньше толщины всей кремниевой структуры. До начала освещения через фототиристоры протекает ток утечки

$$
J_{0}=q n_{k 0} v\left(E_{k 0}\right) S_{k},
$$

где $q-$ элементарный заряд, $n_{k 0}-$ концентрация электронов и дырок в истощенном $i$-слое, поставляемых инжекцией из эмиттеров и термогенерацией в $i$-слое, $E_{k 0}=U_{k 0} / m d, v(E)$ - суммарная дрейфовая скорость 
электронов и дырок, зависящая от напряженности поля $E$ по закону

$$
v(E)=v_{s n} \frac{E}{|E|+E_{s n}}+v_{s p} \frac{E}{|E|+E_{s p}},
$$

$E_{s n, s p} \ll E_{k 0}-$ характерные напряженности поля, выше которых дрейфовые скорости приближаются к величинам $v_{s n, s p} \approx 10^{7} \mathrm{~cm} / \mathrm{s}$. Для определенности будем считать, что каждый фототиристор освещается импульсом света с мощностью (окончательные результаты очень слабо зависят от формы импульса излучения)

$$
P(t)=P_{M} \frac{\sqrt{2 e}}{m} \frac{t}{t_{p h}} \exp \left[-\left(\frac{t}{t_{p h}}\right)^{2}\right],
$$

где $P_{M}-$ пиковая мощность. Энергия $m$ таких импульсов $W_{p h}=\sqrt{e / 2} P_{M} t_{p h}$. После начала освещения усредненная по толщине структуры концентрация $\bar{n}_{k}$ в освещенных областях увеличивается со временем по закону

$$
\begin{gathered}
\bar{n}_{k}(t)=n_{k 0}+n_{k 1}\left\{1-\exp \left[-\left(t / t_{p h}\right)^{2}\right]\right\} \\
n_{k 1}=W_{p h} \frac{1-R_{p h}}{\hbar \omega S_{0}} \frac{1-e^{-\kappa d}}{m d}
\end{gathered}
$$

где $\hbar \omega-$ энергия кванта света, $R_{p h}-$ коэффициент отражения от поверхности окна, $\kappa-$ коэффициент поглощения света в кремнии. Можно показать, что при этом падение напряжения $U_{k}$ на коммутаторе связано с протекающим через нагрузку током $J$ соотношением [11]

$$
C_{k} \frac{d U_{k}}{d t}=J-S_{0} \bar{j}
$$

где $C_{k}=\varepsilon S_{k} / m d-$ емкость коммутатора в блокирующем состоянии, $\varepsilon$ - диэлектрическая проницаемость кремния, $\bar{j}-$ усредненная по толщине $i$-слоя плотность тока электронов и дырок в освещенной области. Строго говоря, для вычисления функции $\bar{j}(t)$ нужно решить уравнение (7) вместе с уравнением Пуассона и уравнениями непрерывности для концентраций электронов и дырок, как это сделано в [11]. Однако в настоящей работе мы используем упрощенную формулу

$$
\bar{j}=q \bar{n}_{k} v\left(E_{k}\right)
$$

которая приближенно верна при $t \ll d / v-$ времени пролета электронов и дырок через $i$-слой, если напряженность поля в $i$-слое слабо зависит от координаты (т.е. $\left.E_{k} \approx U_{k} / m d\right)$ и $\kappa d<1$. Эта упрощенная модель оправдана (точное численное моделирование (см. далее) подтверждает ее применимость) не только потому, что в качественном отношении правильно описывает переключение фототиристоров в проводящее состояние, но и вследствие слабого влияния параметров „почти идеального коммутатора“ на процесс возбуждения ЛПМ. Необходимо подчеркнуть, что сказанное относится только к приборам специальной конструкции $[10,17]$ при надлежащем выборе параметров управляющих импульсов света, но совершенно неприменимо к обычным высоковольтным фототиристорам [18].

Используя законы Кирхгофа для контура и формулы (1) - (8), нетрудно получить следующую систему уравнений и соответствующих им начальных условий:

$$
\begin{gathered}
\frac{d J}{d t}=\frac{Q}{\tau^{2}}-\frac{U_{R}+U_{k}}{\rho \tau}, \quad J(0)=J_{0}, \\
\frac{d Q}{d t}=-J, \quad Q(0)=C U_{C 0}, \\
\frac{d n}{d t}=n\left(N_{\mathrm{Cu}}-n\right) K_{i 0} A\left(U_{R} / \Lambda\right), \quad \eta(0)=0 \\
\frac{d U_{R}}{d t}=R_{0} M \frac{\frac{d J}{d t}-\frac{J}{n} \frac{d n}{d t}}{\frac{n}{n_{0}}-\frac{J R_{0}}{\Lambda} \frac{d M}{d E}}, \quad U_{R}(0)=J_{0} R_{0}, \\
\frac{d U_{k}}{d t}=C_{k}^{-1}\left[J-q \bar{n}_{k} v\left(\frac{U_{k}}{m d}\right) S_{0}\right], \quad U_{k}(0)=U_{k 0},
\end{gathered}
$$

где $\tau=\sqrt{L C}, \rho=\sqrt{L / C}, R_{0}=\Lambda / q \mu_{0} n_{0} S_{l p}, Q$ - заряд накопительной емкости, $U_{C 0}=\left(U_{k 0}+U_{R 0}\right)-$ начальное напряжение на накопительной емкости.

\section{Результаты расчетов и их обсуждение}

В этом разделе в качестве примера изложены результаты решения системы приближенных уравнений (9)-(13) методом Рунге-Кутта и точного численного моделирования процессов в фототиристоре с помощью программы „Исследование“ [19]. Использовались значения параметров ЛПМ и контура, взятые из работы [3]: $\Lambda=48 \mathrm{~cm}, r_{p l}=1 \mathrm{~cm}, N_{\mathrm{Ne}}=2 \cdot 10^{18} \mathrm{~cm}^{-3}$, $N_{\mathrm{Cu}}=1.5 \cdot 10^{-3} \mathrm{~N}_{\mathrm{Ne}}, \quad n_{0}=(2-20) \cdot 10^{13} \mathrm{~cm}^{-3}, \quad$ индуктивность газоразрядной трубки с коаксиальным обратным токопроводом $L=160 \mathrm{H}$ н, накопительная емкость $C=1.5$ нФ заряжена до начального напряжения $U_{C 0}=15 \mathrm{kV}$. Предполагалось, что коммутатор состоит из $m=3$ последовательно соединенных кремниевых фототиристоров со следующими параметрами каждого из них: $S_{k}=1 \mathrm{~cm}^{2}, S_{0}=0.5 S_{k}, d=600 \mu \mathrm{m}$, напряжение пробоя $U_{b}=6.34 \mathrm{kV}$, ток утечки $J_{0}=1.7 \mathrm{~mA}$ при $U_{k 0}=5 \mathrm{kV}$, время восстановления блокирующей способности $10 \mu \mathrm{s}^{1}$. Тиристоры переключаются в проводящее состояние под действием импульсов света с длиной волны $1.064 \mu \mathrm{m}$ (коэффициент поглощения в кремнии $\kappa \approx 32 \mathrm{~cm}^{-1}$ при рабочей температуре кристалла $\left.100^{\circ} \mathrm{C}\right)$.

\footnotetext{
1 Для этого необходимо уменьшить время жизни неравновесных носителей заряда $\tau$ примерно до $1 \mu \mathrm{s}$. При таких $\tau$ обычные высоковольтные фототиристоры [18] неработоспособны из-за низкой эффективности инжекционного механизма модуляции проводимости. Однако при почти однородной по объему кристалла фотоионизации и длительности импульса тока $<0.1 \mu$ s уменьшение $\tau$ до $1 \mu$ s практически не влияет на импульсные характеристики фототиристора, но обеспечивает его работоспособность при частотах вплоть до десятков килогерц.
} 


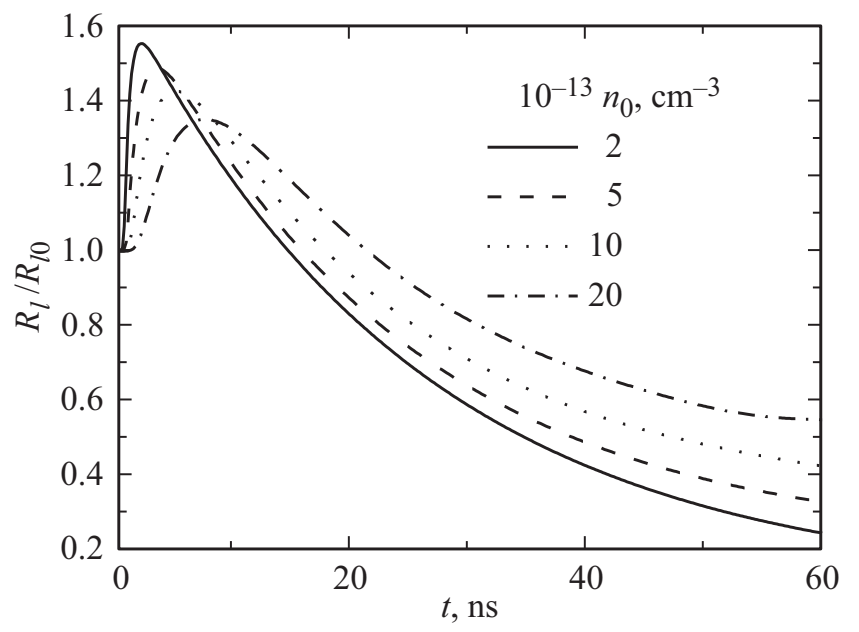

Рис. 3. Зависимости активной составляющей импеданса лазеpa $R_{l}$ от времени, полученные путем решения системы приближенных уравнений (9)-(13) при $W_{p h}=3 \cdot 108 \mu \mathrm{J}, t_{p h}=5 \mathrm{~ns}$ и различных предымпульсных концентрациях электронов $n_{0}$.
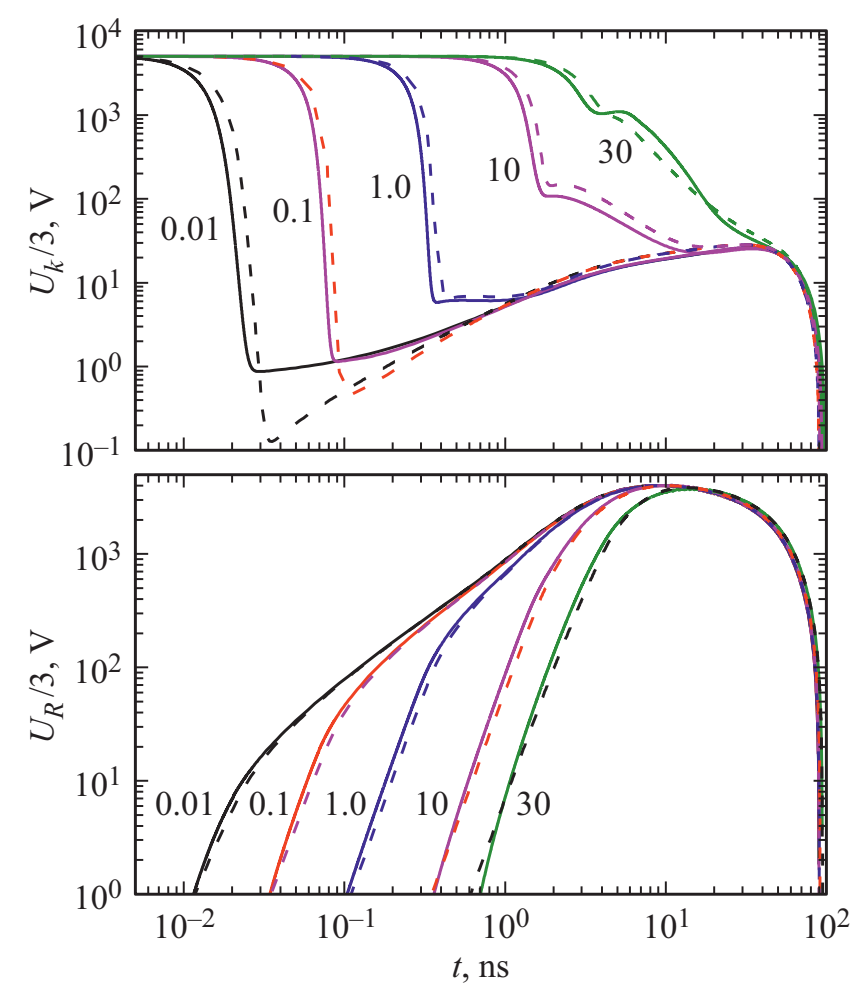

Рис. 4. Зависимости напряжений на коммутаторе $U_{k}$ и на активной составляющей импеданса лазера $U_{R}$ от времени, полученные путем точного численного моделирования (сплошные линии) и решения системы уравнений (9)-(13) (штриховые линии) при $W_{p h}=3 \cdot 122 \mu \mathrm{J}$ и $n_{0}=10^{14} \mathrm{~cm}^{-3}$. Цифры у кривых означают длительность управляющего импульса света $t_{p h}$ в наносекундах.

Результаты расчетов приведены на рис. 3-5. Зависимости $R(t)$, примеры которых изображены на рис. 3 , использовались в программе „Исследование“ [19] для точного моделирования процессов в тиристорных $n^{++}-p^{+}-p-n-n^{+}-p^{++}$-структурах, основные параметры которых приведены выше, а реалистичное распределение легирующих примесей по толщине кристаллов описано в [12]. Полученные таким образом „приближенные“ и „точные“ зависимости $U_{k}(t)$ и $U_{R}(t)$ хорошо согласуются между собой (рис. 4). Заметное относительное расхождение наблюдается только при очень малых (порядка $1 \mathrm{~V}$ ) значениях $U_{k}(t)$. Причина этого, очевидно, состоит в том, что упрощенная модель тиристоров не учитывает падения напряжения (около $0.7 \mathrm{~V}$ ) на прямо смещенных эмиттерных переходах. Однако такое расхождение практически не влияет на результаты расчетов потерь энергии в тиристорах $W_{k}$ (рис. 5). Как видно, $W_{k}$ начинает резко увеличиваться с ростом длительности управляющего импульса света при $t_{p h}>10 \mathrm{~ns}$. Поэтому для управления фототиристорами следует использовать импульсы света с $t_{p h} \leq 5 \mathrm{~ns}$, обеспечивающие переключение в проводящее состояние за время порядка $1 \mathrm{~ns}$ или меньше.

Энергия потерь в тиристорах $W_{k}$ уменьшается с ростом $W_{p h}$, но одновременно увеличиваются потери в управляющем лазере. В результате получается немонотонная зависимость суммарной энергии потерь в оптопаре $W_{\text {sum }}$ от $W_{p h}$, которая имеет минимум (рис. 6). Таким образом, существует оптимальное значение $W_{p h}$, которое в нашем конкретном случае равно $0.33 \mathrm{~mJ}$ при $t_{p h}=5 \mathrm{~ns}$ и КПД управляющего лазера $10 \%$, когда $W_{\text {sum }} \approx 2 W_{k} \approx 6.6 \mathrm{~mJ}$. Так как запасенная в накопительной емкости $C$ энергия $C U_{0}^{2} / 2=169 \mathrm{~mJ}$, то без учета потерь в цепи заряда получается КПД схемы возбуждения более 96\%. Импульсный разогрев тиристоров за один цикл возбуждения примерно равен 0.01 К, так что фактически они работают в режиме постоянного рассеивания средней мощности $P_{k}=W_{k} f$. При $f=20 \mathrm{kHz}$ каждый

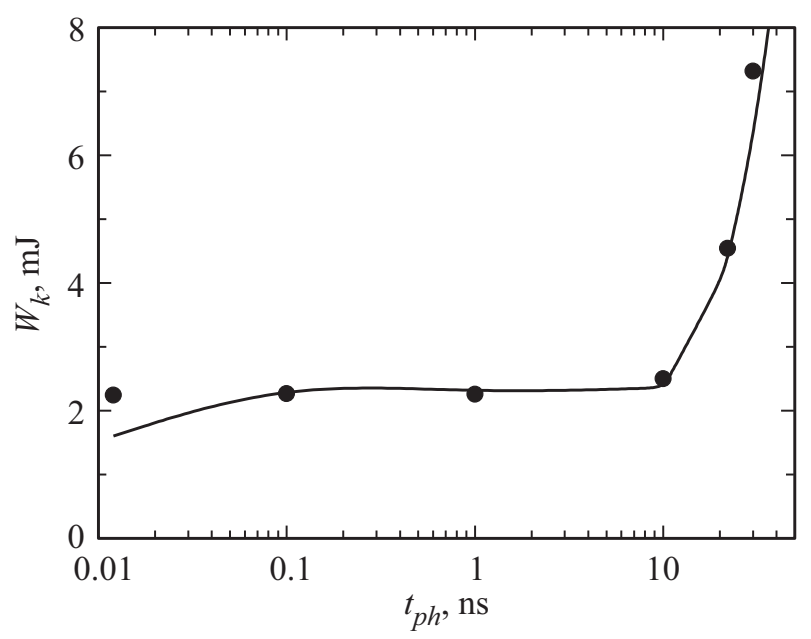

Рис. 5. Зависимости энергии коммутационных потерь в трех фототиристорах $W_{k}$ от длительности управляющего импульса света $t_{p h}$, полученные путем точного численного моделирования (символы) и путем решения системы приближенных уравнений (9)-(13) (линия) при $W_{p h}=3 \cdot 200 \mu \mathrm{J}$ и $n_{0}=10^{14} \mathrm{~cm}^{-3}$. 
из трех тиристоров будет рассеивать среднюю мощность $P_{k} / m \approx 22 \mathrm{~W}$, поэтому для поддержания рабочей температуры кристаллов $100^{\circ} \mathrm{C}$ достаточно использовать простую систему охлаждения с тепловым сопротивлением кристалл-среда $4 \mathrm{~K} / \mathrm{W}$.

Форма и амплитуда импульсов тока $J(t)$ контура и напряжения $U_{R}(t)$ на активной составляющей импеданса ЛПМ остаются практически постоянными при изменении длительности и энергии управляющих импульсов света в разумных пределах, но существенно зависят от предымпульсной концентрации электронов $n_{0}$. Вследствие этого изменяется зависимость мощности $P_{R}=U_{R} J$ от времени и энергия $W_{R}$, поглощаемая ЛПМ на начальном этапе возбуждения, как это изображено на рис. 7,8 . Кроме этого, наблюдается обратное влияние ЛПМ на коммутатор: с ростом $n_{0}$ увеличиваются потери энергии в фототиристорах (рис. 8).

В рассмотренном нами примере контур возбуждения оказался почти точно согласованным с нагрузкой: амплитуда отраженного импульса была примерно в 10 раз меньше, чем основного. Однако на практика такого согласования удается достичь далеко не всегда, и возникновение мощного отраженного импульса приводит к дополнительному ужесточению режима работы тиратронов $[1,5]$. В связи с этим следует отметить еще одно достоинство описанного коммутатора: фототиристоры способны пропускать большие обратные токи в течение сотен наносекунд безо всякого для себя вреда. Разумеется, при этом увеличиваются потери, но незначительное увеличение энергии управляющего импульса света позволяет полностью скомпенсировать этот эффект. Поэто-

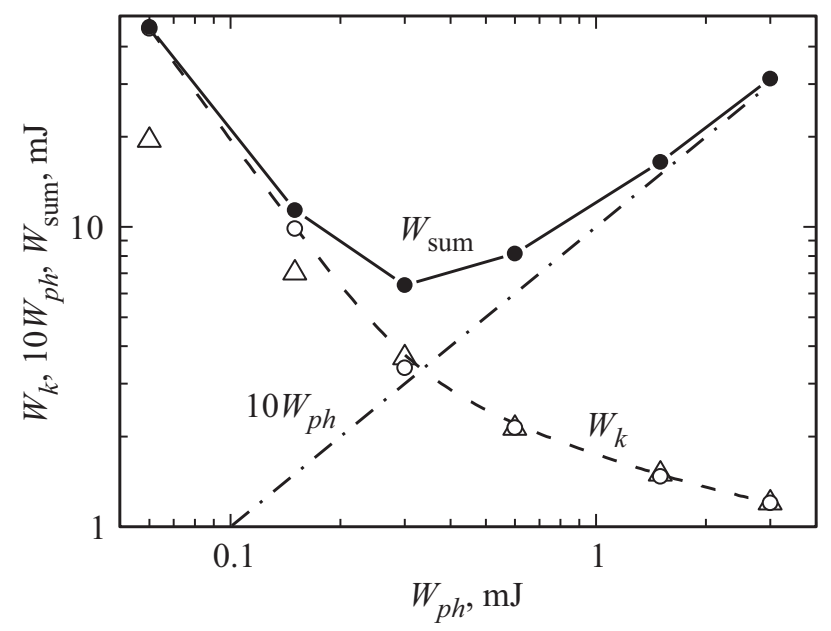

Рис. 6. Зависимости энергии, рассеиваемой тремя тиристорами коммутатора $W_{k}$, полученые путем точного численного моделирования (светлые кружки + штриховая линия) и путем решения системы приближенных уравнений (9)-(13) (светлые треугольники)), источником питания управляющего лазера с КПД $10 \%\left(10 W_{p h}\right.$, штрихпунктир) и суммарной энергии потерь оптопары $W_{\text {sum }}$ за один импульс возбуждения (темные кружки + сплошная линия) от энергии $W_{p h}$ импульса излучения управляющего лазера при $t_{p h}=5 \mathrm{~ns}$ и $n_{0}=10^{14} \mathrm{~cm}^{-3}$.

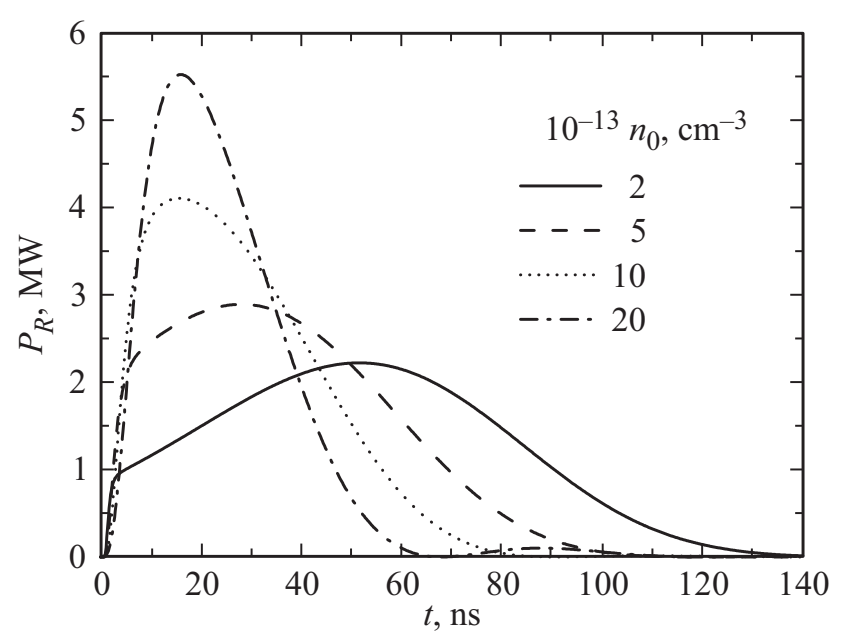

Рис. 7. Зависимости мощности $P_{R}$, рассеиваемой активной составляющей импеданса лазера $R_{l}$ от времени, полученные путем решения системы приближенных уравнений (9)-(13) при $W_{p h}=3 \cdot 108 \mu \mathrm{J}, t_{p h}=5 \mathrm{~ns}$ и различных предымпульсных концентрациях электронов $n_{0}$.

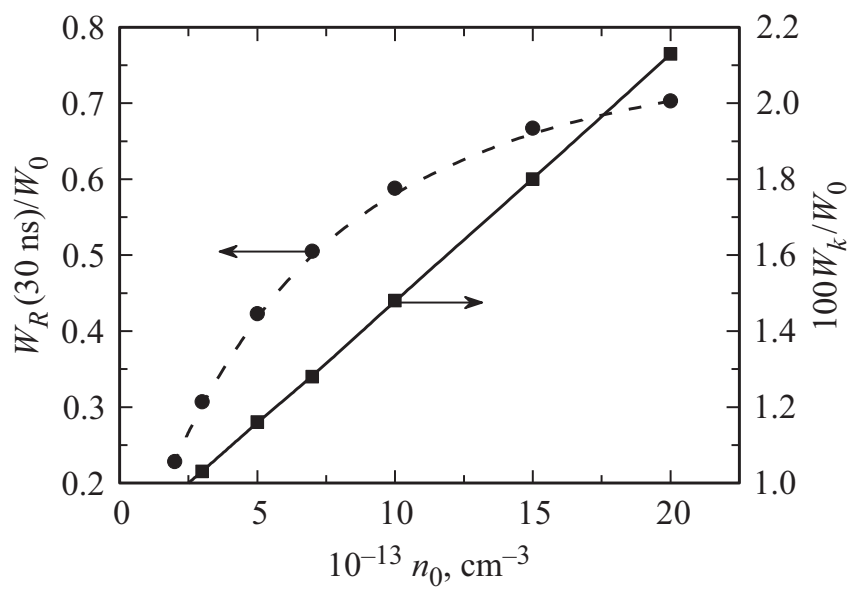

Рис. 8. Зависимости нормированных значений энергии $W_{R}$, рассеиваемой активной составляющей импеданса лазера $R_{l}$ за $30 \mathrm{~ns}$ после начала импульса возбуждения, и энергии $W_{k}$, рассеиваемой коммутатором, от предымпульсной концентрациии электронов $n_{0}$, полученные путем решения системы приближенных уравнений (9)-(13) при $W_{p h}=3 \cdot 108 \mu \mathrm{J}$ и $t_{p h}=5 \mathrm{~ns}$.

му фототиристоры можно использовать почти столь же эффективно даже для возбуждения коаксиальных ЛПМ импульсно-периодическим индукционным разрядом [20], который невозможно реализовать при использовании тиратронов.

\section{Заключение}

В заключение отметим три проблемы, связанные с возможностью применения описанного коммутатора. Первая из них - сложность создания малоиндуктивной сборки последовательно соединенных фототиристо- 
ров [9] и отсутствие промышленной технологии изготовления кремниевых структур с напряжением пробоя $U_{b}$ более $10 \mathrm{kV}$ [18]. В принципе ее можно решить, используя карбид-кремниевые фотитиристоры, так как уже созданы опытные образцы биполярных ключей на основе $4 \mathrm{H}-\mathrm{SiC}$ с $U_{b}=27 \mathrm{kV}$ [21]. Однако промышленная технологии подобных приборов также еще не освоена, a управляющие лазеры с подходящей длиной волны излучения $\lambda=375 \mathrm{~nm}$ труднодоступны. Поэтому в настоящее время описанный коммутатор можно применять для возбуждения либо не очень мощных ЛПМ с короткой газоразрядной трубкой, либо многосекционных ЛПМ [22,23] и ЛПМ с поперечным разрядом [24,25]. Вторая проблема - высокая стоимость волоконных лазеров, которая может решиться в ходе дальнейшего прогресса технологии импульсных полупроводниковых лазеров. Третья проблема состоит в том, что изложенные выше результаты, иллюстрирующие достоинства оптопары „волоконный лазер-фототиристор“, не позволяют ответить на вопрос о том, насколько таким способом можно улучшить выходные оптические характеристики ЛПМ - среднюю мощность излучения и полный практический КПД. Для этого необходимо решить задачу, выходящую за рамки настоящей работы. А именно, надо провести оптимизацию режима работы ЛМП с использованием одной из полномасштабных моделей [1], заменив в ней феноменологическое описание тиратрона (см. раздел 8.4 в [1]) на весьма точное и физически обоснованное уравнение (13).

Автор благодарен С.Н. Юркову и Т.Т. Мнацаканову, предоставившим возможность провести моделирование процесса коммутации с помощью программы „Исследование“, Н.М. Лепехину и М.М. Маликову за плодотворное обсуждение ряда вопросов, затронутых в настоящей работе. Работа выполнена при поддержке РФФИ (грант № 16-08-01292).

\section{Список литературы}

[1] Лазеры на самоограниченных переходах атомов металлов2. Т. 1. / Под ред. Батенина В.М. М.: ФИЗМАТЛИТ, 2009. $544 \mathrm{c}$.

[2] Григорьяни, А.Г., Казарян М.А., Лябин Н.А. Лазеры на парах меди: конструкция, характеристики и применения. М.: ФИЗМАТЛИТ, 2005. 312 с.

[3] Бохан П.А., Гугин П.П., Закревский Дм.Э., Лаврухин М.А., Казарян М.А., Лябин Н.А. // Квант. электрон. 2013. Т. 43. № 8. C. 715-719.

[4] Фогельсон Т.Б., Бреусова Л.Н., Вагин Л.Н. Импульсные водородные тиратроны. М.: Сов. радио, 1974. 212 с.

[5] Исаев А.А., Леммерман Г.Ю. // Труды ФИАН. 1987. Т. 181. C. $164-179$.

[6] Бохан П.А., Закревский Дм.Э., Лаврухин М.А., Лябин Н.А., Чурсин А.Д. // Квант. электрон. 2016. Т. 46. № 2. C. $100-105$.

[7] Бохан П.А., Гугин П.П., Закревский Дм.Э., Лаврухин М.А. // Письма в ЖТФ. 2012. Т. 38. Вып. 8. С. 63-70.
[8] Бохан П.А., Гугин П.П., Закревский Дм.Э., Лаврухин М.А. // Письма в ЖТФ. 2013. Т. 39. Вып. 17. С. 44-52.

[9] Александров В.М., Бужинский О.И., Грехов И.В. и др. // Квант. электрон. 1981. Т. 86. № 1. С. 191-193.

[10] Воле В.М., Воронков В.М., Грехов И.В. и др. // ЖТФ. 1981. Т. 51. Вып. 2. С. 373-379.

[11] Кюрегян А.С. // ФТП. 2014. Т. 48. Вып. 12. С. 1686-1692.

[12] Кюрегян А.С. // ФТП. 2017. Т. 51. Вып. 9. С. 1257-1262. doi 10.21883/FTP.2017.09.44891.8494

[13] Кюрегян А.С. // ФТП. 2017. Т. 51. Вып. 9. С. 1263-1266. doi 10.21883/FTP.2017.09.44892.8495

[14] Кюрегян А.С. // ФТП. 2019. Т. 53. Вып. 4. С. 528-532.

[15] http://www.ipgphotonics.com/ru/products/lasers

[16] Мнацаканян А.Х., Найдис Г.В., Штернов Н.П. // Квант. электрон. 1978. Т. 5. Вып. 3. С. 597-602.

[17] Glidden S.C., Sanders H.D. // US Patent N 8,461,620 B2 (2013).

[18] http://www.elvpr.ru/poluprovodnikprib/tiristory/flyers/ /TL_2017.pdf

[19] Mnatsakanov T.T., Rostovtsev I.L., Philatov N.I. // Solid-State Electronics. 1987. V. 30. N 6. P. 579-585. doi 10.1016/00381101(87)90215-2

[20] Батенин В.М., Казарян М.А., Карпухин В.T., Лябин Н.А., Маликов М.М., Сачков В.И. // Оптика атмосферы и океана. 2016. Т. 29. В. 2. С. 112-120. doi 10.15372/AOO20160205

[21] Brunt E., Cheng L., O'Loughlin M.J. et al. // Materials Science Forum. 2015. V. 821-823. P. 847-850. doi 10.4028/www.scientific.net/MSF.821-823.847

[22] Pack J.L., Liu C.S., Feldman D.W., Weaver L.A. // Rev. Sci. Instrum. 1977. V. 48. N 1047. doi 10.1063/1.1135181

[23] Кирилов А.Е., Кухарев В.Н., Солдатов А.Н. // Квант. электрон. 1979. Т. 6. № 3. С. 473-477.

[24] Piper J.A. // IEEE J. Quantum Electron. 1978. V. QE-14. N 6. P. 405-407.

[25] Соколов А.В., Свиридов А.В. // Квант. электрон. 1981. Т. 8. № 8. C. $1686-1696$. 